\section{Phaeochromocytoma presenting as acute hyperamylasaemia and multiple organ failure}

Phaeochromocytoma may present in many different ways. We report an unusual presentation of phaeochromocytoma in $a$ man with hyperamylasaemia and multiple organ failure thought to be due to acute relapsing pancreatitis. Abdominal ultrasound and computerised tomography (CT) examinations revealed a mass at the tail of the pancreas. Fine needle biopsy of the mass precipitated headache, intense vasoconstriction and labile blood pressure. He proceeded to laparotomy, at which an 8 $\times 9 \mathrm{~cm}$ mass was found to be replacing the left adrenal gland. Histological examination revealed a phaeochromocytoma. This case illustrates that hyperamylasaemia and multiple organ failure may be unusual presentations of phaeochromocytoma and phaeochromocytoma should be considered in the differential diagnosis of a peripancreatic mass found by ultrasound or CT.

Le phéochromocytome peut se révéler de différentes façons. Nous décrivons une présentation inhabituelle de phéochromocytome chez un homme souffrant d'amylasémie et de défaillance multiviscérale imputées à une pancréatite récidivante. Lultrasonographie abdominale et la tomodensitométrie ont décelé une tumeur de la queue du pancréas. Une biopsie à l'aiguille fine a déclenché des céphalées, une vasoconstriction intense et de la labilité tensionnelle. Le patient est laparatomisé et on découvre une masse de $8 \times 9 \mathrm{~cm}$ à la place de la surrénale gauche. L'examen histologique révèle un phéochromocytome. Cette observation illustre que le phéochromocytome peut se manifester inhabituellement par de l'hyperamylasémie et une défaillance multiviscérale et que le phéochromocytome doit être inclus dans le diagnostic différentiel d'une masse péripancréatique trouvée à l'ultrasonographie ou à la tomodensitométrie.

\section{Key words}

SURGERY: phaeochromocytoma.

From the Intensive Care Unit* and the Departments of Medicine $\dagger$ and Surgery $\ddagger$, The Middlesex Hospital, Mortimer Street, London, England WIN 8AA.

Address correspondence to: Dr. T. J. Gan, Department of Anesthesiology, Duke University Medical Center, Box 3094, Durham, NC 27710, U.S.A.

Accepted for publication 6th November, 1993.
Phaeochromocytoma is an uncommon tumour of the adrenal medulla derived embryologically from neuroectodermal cells. It secretes catecholamines, an action which accounts for its interesting and often bizarre clinical signs and symptoms. Although the majority of patients present with sustained or paroxysmal hypertension, the incidence of phaeochromocytoma in hypertensive patients is less than $1 \%{ }^{1,2}$ We report a case of phaeochromocytoma presenting as acute hyperamylasaemia and multiple organ failure.

\section{Case report}

A 58-yr-old Caucasian man with a diagnosis of acute relapsing pancreatitis was referred to this hospital for the investigation of a mass in the tail of the pancreas. Two months previously he had presented to his local hospital with abdominal pain and fever of sudden onset. He denied excess alcohol intake and was receiving no drug treatment. Nine years previously he had been investigated for alternating abdominal pain and headaches; symptoms had resolved spontaneously and all investigations were negative. On examination he was normotensive (BP: 110/ 75) and had diffuse abdominal pain. Initial laboratory investigations yielded the following results: haemoglobin $15.5 \mathrm{~g} \cdot \mathrm{dl}^{-1}$, haematocrit $45 \%$, platelet count 185,000 $\mu l^{-1}$, serum sodium 142 , potassium 4.8 , calcium 2.15 , glucose 6.3 , urea $5.6 \mathrm{mmol} \cdot \mathrm{L}^{-1}$, creatinine 105 $\mu \mathrm{mol} \cdot \mathrm{L}^{-1}$ (normal 55-100 $\mu \mathrm{mol} \cdot \mathrm{L}^{-1}$ ). The serum amylase concentration was elevated at $4308 \mathrm{IU} \cdot \mathrm{L}^{-1}$. A diagnosis of acute pancreatitis was made. The patient subsequently became hyperglycaemic and hypoxaemic. Serum glucose $15 \mathrm{mmol} \cdot \mathrm{L}^{-1}$, arterial blood gases: $\mathrm{pH}$ $=7.0 \mathrm{PCO}_{2}=6.9 \mathrm{kPa}, \mathrm{PO}_{2}=8.1 \mathrm{kPa}$, bicarbonate $12.2 \mathrm{mmol} \cdot \mathrm{L}^{-1}, \mathrm{FIO}_{2}=0.6$ on a fixed performance mask. In addition the chest $x$-ray showed diffuse bilateral infiltration sparing the bases. Pulmonary artery catheter insertion revealed a capillary wedge pressure of 12 $\mathrm{mmHg}$, compatible with adult respiratory distress syndrome (ARDS). Mechanical ventilation was commenced and continued for 11 days. On the third day, the patient developed disseminated intravascular coagulopathy (DIC), haemoglobin $9.8 \mathrm{~g} \cdot \mathrm{dl}^{-1}$, white cell count 
$18,000 \cdot \mu \mathrm{l}^{-1}$, platelet count $35,000 \cdot \mu \mathrm{l}^{-1}$, prolonged prothrombin and partial thromboplastin times with increased fibrin degradation products and low fibrinogen level. Acute renal failure ensued (urinary sodium $75 \mathrm{mEq} \cdot \mathrm{L}^{-1}$, urinary specific gravity 1.010, urinary microscopy showed 45 erythrocytes per visual field, protein and waxy casts, serum urea $45 \mathrm{mmol} \cdot \mathrm{L}^{-1}$ and creatinine $530 \mu \mathrm{mol} \cdot \mathrm{L}^{-1}$ ) which required haemofiltration. Blood pressure fluctuated between $170 / 100$ and $80 / 65 \mathrm{mmHg}$, the hypotensive episodes responded to colloid administrations. Following his recovery an abdominal ultrasound was performed which showed no stones in the biliary tract or gall bladder; the head and body of the pancreas were normal, but a 7 $\times 7 \mathrm{~cm}$ mass of mixed echogenicity was detected in the tail of the pancreas.

He was discharged and two weeks later presented again with a sudden onset of severe epigastric pain, radiating through to the back, and vomiting. Again he was normotensive (BP:120/75) and serum amylase was 4235 $\mathrm{IU} \cdot \mathrm{L}^{-1}$. Acute respiratory failure rapidly followed with clinical and radiological pictures similar to the first episode. Renal function was mildly abnormal but did not require renal support therapy. Blood pressure range was similar to the previous episode. The patient was treated with mechanical ventilation for four days, after which he recovered. A repeat ultrasound scan revealed the same $7 \times 7 \mathrm{~cm}$ mass in the tail of the pancreas and no other abnormalities were detected.

In view of these recurrent episodes of multiple organ failure and still without a satisfactory explanation, he was referred to this hospital for further investigation. On admission, the patient was well, there were no abdominal signs and he was normotensive. Preliminary investigations (full blood count, urea and electrolytes, liver function tests and serum amylase) were all normal.

Endoscopic retrograde cholangio-pancreatography was performed and was reported as normal. An abdominal ultrasound and CT scan confirmed a mass in the tail of the pancreas: the head of the pancreas appeared normal. The results of these investigations were thought not to be in keeping with the recent history of recurrent acute pancreatitis and a pancreatic tumour was suspected. In view of this an ultrasound guided percutaneous needle biopsy of the mass was performed. He was normotensive before the procedure but during it, the patient became unwell with a labile blood pressure ranging from 230 / 130 to $40 / 20 \mathrm{mmHg}$ accompanied by profuse sweating and vasoconstriction. He was transferred to the intensive care unit. Radial artery and pulmonary artery catheters were inserted for continuous monitoring. Arterial blood analysis was normal breathing room air and the cardiac index was low at $1.7 \mathrm{~L} \cdot \mathrm{min}^{-1} \cdot \mathrm{m}^{-2}$, with a right atrial pressure of $5 \mathrm{mmHg}$ and pulmonary capillary wedge pressure of $11 \mathrm{mmHg}$. An ECG showed T-wave inversion in the lateral chest leads. The possibility of phaeochromocytoma was raised. A 24-hr urinary collection for VMA was commenced and was found to be markedly elevated at $351 \mu \mathrm{mol} \cdot 24 \mathrm{hr}^{-1}$ (normal $<32 \mu \mathrm{mol} \cdot 24 \mathrm{hr}^{-1}$ ). The patient was treated with an intravenous infusion of phentolamine and labetalol for control of the hypertension. Numerous episodes of acute hypotension developed which responded to colloid and ephedrine therapy. The blood pressure stabilised over the next two days with therapy and surgery to remove the adrenal tumour was carried out on the third day.

Monitoring (which included ECG, pulse oximetry, radial arterial, central venous and pulmonary artery pressures) were established before the induction of anaesthesia. General anaesthesia was induced with fentanyl 3 $\mu \mathrm{g} \cdot \mathrm{kg}^{-1}$ followed by etomidate and vecuronium $\dot{i}$. Sodium nitroprusside (SNP) and nitroglycerin (GTN) infusions were employed to control hypertensive episodes during any manipulation of the tumour and the maximum rates of infusion of SNP and GTN were 15 and $20 \mu \mathrm{g} \cdot \mathrm{kg}^{-1} \cdot \mathrm{hr}^{-1}$ respectively. One episode of ventricular tachycardia was treated successfully with lignocaine $i v$ given as a bolus and followed by continuous infusion.

At operation, a $9 \times 8 \mathrm{~cm}$ vascular mass was found above the upper pole of the left kidney replacing the adrenal gland but the body and the tail of the pancreas were normal. A left adrenalectomy was performed and histological examination of the adrenal mass revealed a phaeochromocytoma comprised of regular polygonal cells with granular eosinophilic cytoplasm. There was extensive haemorrhage and necrosis. Immuno-histochemical studies with neuroendocrine markers were positive for neurone specific enolase, chromogranin and neurofilament.

Postoperatively, the patient was transferred to the intensive care unit and SNP and GTN infusions were required for only three hours after surgery. On the second postoperative day his urea and creatinine concentrations were rising (urea $25 \mathrm{mmol} \cdot \mathrm{L}^{-1}$, creatinine 330 $\mu \mathrm{mol} \cdot \mathrm{L}^{-1}$ ) and haemofiltration was commenced for 36 hr. A repeat ECG showed that the T-waves had reverted to normal. The patient's subsequent recovery was uneventful and he was discharged with normal renal and cardiac function. He remains well 21 mo after surgery.

\section{Discussion}

Phaeochromocytoma has extremely variable presenting features. In the majority of patients there is continuous secretion of an excess of catecholamines, mainly noradrenaline. This usually results in sustained mild to moderate hypertension. In addition, there are paroxysmal peaks of catecholamine secretion, which usually occur 
spontaneously but may be provoked by movement of the trunk or by abdominal palpation. ${ }^{1-3}$ These catecholamine crises result in headache, palpitations, sweating, nausea, tremor, extreme pallor (due to vasoconstriction) and anterior chest and upper abdominal pain with a feeling of impending doom (angor animi). ${ }^{1-3}$ Hypertension often exceeds $250 / 150 \mathrm{mmHg}$, but may be followed by periods of hypotension. These hypertensive episodes may occur several times a day or be separated by several weeks. In a small proportion of patients the blood pressure may be normal between paroxysms. ${ }^{1-3}$

Our patient had presented with abdominal pain and headaches nine years previously. These symptoms may have been attributable to a phaeochromocytoma but he was reportedly normotensive then. He subsequently presented on two occasions with a sudden onset of severe abdominal pain and hyperamylasaemia. On both occasions respiratory failure requiring mechanical ventilation resulted, and on the first occasion the clinical course was complicated by DIC and acute renal failure.

Multiple organ failure caused by phaeochromocytoma is rare. Newell et al. ${ }^{4}$ summarised three of the 27 patients with phaeochromocytoma over a period of $14 \mathrm{yr}$ had features of multiple organ failure, with encephalopathy, arterial hyper- and hypotension, renal insufficiency and respiratory failure. One of the patients died during attempts to find the source of sepsis and the other two were successfully treated with prompt diagnosis, vigorous medical therapy and urgent tumour removal. Bergland ${ }^{5}$ reviewed 539 cases of phaeochromocytoma and found 12 presenting with shock at initial evaluation. The effects of catecholamines on organ function have been studied in various animals and human models ${ }^{5}$ using catecholamine infusions in which shock and intravascular volume depletion developed rapidly and persisted for hours following cessation of the infusion of catecholamines.

In our patient the two episodes of acute respiratory failure with the characteristic radiographic changes, and of high normal pulmonary capillary wedge pressure were compatible with ARDS. The detailed pathogenesis of ARDS caused by phaeochromocytoma is not completely understood. It is hypothesized that the excessive release of catecholamines may result in a toxic effect on the pulmonary capillaries. Colice et al. ${ }^{6}$ demonstrated that noncardiogenic lung oedema may be produced by the highdose catecholamine infusions.

Our patient received haemofiltration for acute renal failure on two occasions. The acute tubular necrosis was most likely secondary to ischaemic damage caused by either intense vasoconstriction or hypovolaemia induced by the out-pouring of catecholamines.

Phaeochromocytoma presenting as hyperamylasaemia is uncommon. Yamanishi et al. ${ }^{7}$ described the case of a 62-yr-old woman who presented with abdominal pain, nausea, headache and palpitations. The patient had a tenyear history of episodic hypertension. On admission the serum amylase was elevated at $2294 \mathrm{IU} \cdot \mathrm{L}^{-1}$. The patient was thought to have acute pancreatitis. Conservative treatment was successful initially but the patient presented with further episodes of abdominal pain, nausea, vomiting and hypertension. An abdominal CT scan subsequently revealed a left supra-renal tumour. Before surgery (at which a phaeochromocytoma was removed) the patient experienced two further episodes of abdominal pain and hyperamylasaemia, one provoked by a barium meal study, the other by exercise.

The cardiac manifestations of phaeochromocytomas may include tachycardias, arrhythmias, myocardial ischaemia and congestive cardiac failure. ${ }^{8}$ The T-wave changes and ventricular arrhythmias may be caused by intense vasoconstriction from catecholamine release resulting in hypoperfusion. Van Vliet et al. ${ }^{9}$ examined 26 fatal cases of phaeochromocytoma and found histological evidence of "acute catecholamine myocarditis" with focal degeneration, necrosis and oedema of myocardial cells, and left ventricular hypertrophy at autopsy. Our patient underwent further cardiac investigation (ECG and echocardiography) following the recovery of the illness and no abnormality was found.

Headache and labile blood pressure are extremely unusual responses to fine needle aspiration of a pancreatic mass and alerted us to the possibility of a phaeochromocytoma. It is rare for a pancreatic tumour to present with acute recurrent hyperamylasaemia and this together with the ultrasound and CT scan showing a normal pancreatic head might have alerted us to an alternative diagnosis. Percutaneous fine-needle biopsy of a phaeochromocytoma is usually not attempted when the diagnosis is suspected clinically or biochemically for fear of inducing a hypertensive crisis. However, only $50 \%$ of the patients with phaeochromocytomas have paroxysmal hypertensive episodes and at least $14 \%$ may have atypical or the absence of clinical signs. ${ }^{10}$ Hence, the possible diagnosis of phaeochromocytoma remains an important consideration for radiologists performing percutaneous needle biopsy of adrenal masses. Casola et al. ${ }^{10}$ reported four cases of phaeochromocytomas diagnosed incidentally when the patients underwent percutaneous ultrasound or CT guided biopsies. No patient had any symptoms suggestive of phaeochromocytoma. Three patients were normotensive and one had mild hypertension (160/ $90 \mathrm{mmHg}$ ). Indeed in two of the patients biochemical evaluations had been obtained which showed normal values for urinary catecholamines and VMA.

In summary, this case illustrates two points, namely that hyperamylasaemia and multiple organ failure are un- 
usual presentations of phaeochromocytomas and secondly the possibility of a phaeochromocytoma needs to be entertained in the differential diagnosis of a peripancreatic mass before biospy or surgical exploration.

\section{References}

1 Hull CJ. Phaeochromocytoma. Diagnosis, pre-operative preparation and anaesthetic management. $\mathrm{Br} \mathrm{J}$ Anaesth 1986; 58: 1453-68.

2 Van Heerden JA, Sheps SG, Hamberger B, Sheedy PF, Poston JG, Re Mine WH. Phaeochromocytoma: current status and changing trends. Surgery 1982; 91: 367-73.

3 Bravo EL, Gifford RW. Current concepts. Pheochromocytoma: diagnosis, localization and management. $\mathrm{N}$ Engl $\mathrm{J}$ Med 1984; 311: 1298-303.

4 Newell K, Prinz RA, Picleman J, et al. Phaeochromocytoma multisystem crisis. Arch Surg 1988; 123: 956-9.

5 Bergland BE. Pheochromocytoma presenting as shock. Am J Emerg Med 1989; 7: 44-8.

6 Colice GL, Maatthay MAA, Bass E, Matthay RA. Neurogenic pulmonary edema. Am Rev Resp Dis 1984; 130: 941-8.

7 Yamanishi J, Nishikawa M, Ohomori Y, Furuta $Y$, Fukuzaki $H$. Phaeochromocytoma with transient hyperamylasaemia during hypertensive crisis. BMJ 1985; 291: 1171.

8 Hickler RB, Thorn GW. Pheochromocytoma. In: Thorn GW, Adam RD, Braunwald E, Isselbacher KJ, Petersdorf RG (Eds.). Harrison's Principles of Internal Medicine. 8th ed. New York: McGraw-Hill, 1977; 557-63.

9 Van Vliet PD, Burchell HB, Titus JL. Focal myocarditis associated with pheochromocytoma. New Engl J Med 1966; 274: 1102-8.

10 Casola $G$, Nicolet $V$, van Sonnenberg E, et al. Unsuspected pheochromocytoma: risk of blood-pressure alterations during percutaneous adrenal biopsy. Radiology 1986; 159: 733-5. 